\title{
L-2-Hydroxyglutaric Aciduria - a Rare Type of Organic Aciduria Presenting as Seizures and Developmental Delay in a Filipino Child
}

\author{
Cristine P. Lopez, Sheryl V. Decena, Kathleen Gayl D. Fonacier and Mary Anne D. Chiong \\ Biochemical Genetics Laboratory, Institute of Human Genetics, National Institutes of Health, University of the Philippines Manila
}

\begin{abstract}
L-2-hydroxyglutaric aciduria (L-2-HGA) is a rare, autosomal recessive organic aciduria with increased levels of L-2hydroxyglutaric acid in the urine and other body fluids. Clinical presentation includes developmental delay, epilepsy, and typical neuroimaging findings.

This is a report of the clinical, neuroimaging, and biochemical findings of the first diagnosed case of L-2-hydroxyglutaric aciduria in the Philippines. This paper likewise reaffirms the importance of locally available biochemical tests in diagnosing inborn error of metabolism.
\end{abstract}

Key Words: L-2-hydroxyglutaric aciduria, organic aciduria, developmental delay, seizures, developmental regression

\section{Introduction}

L-2-hydroxyglutaric aciduria (L-2-HGA) is a rare neurodegenerative metabolic disorder that follows an autosomal recessive mode of inheritance. It is characterized by elevated levels of L-2-hydroxyglutaric aciduria in urine, plasma, and cerebrospinal fluid (CSF). ${ }^{1,2}$ Clinical manifestations of the condition include speech and mental developmental delay, pyramidal and extrapyramidal signs, demyelination of white matter, cerebellar dysfunctions, epilepsy, macrocephaly, and other neurologic symptoms..$^{2,3,4}$ In some patients, brain tumor formation was also reported. 5,6 Typically the disorder presents in infancy but there are reported adult cases with a milder phenotype. ${ }^{7,8}$ The diagnosis of L-2-HGA is established by urine organic acid screening of 2-hydroxyglutaric acid and following chiral differentiation. The estimated prevalence and incidence of L2-HGA are unknown but it has been reported to affect 140 cases worldwide. ${ }^{9}$

In this report, we present the clinical, biochemical, and neuroimaging findings of the first case of L-2hydroxyglutaric aciduria in the Philippines.

\footnotetext{
Corresponding author: Cristine P. Lopez

Biochemical Genetics Laboratory

Institute of Human Genetics

National Institutes of Health

University of the Philippines Manila

623 Pedro Gil St., Ermita, Manila 1000 Philippines

Telephone: +632 5261725 local 114

Email: cplopez1@up.edu.ph
}

\section{Case Report}

This female patient was 5 years of age at the time of evaluation. On review of history, she is the first child of healthy, non-consanguineous Filipino parents. The maternal and neonatal histories were unremarkable. Her development was normal during the first year of life. At around 1 year of age, she had generalized tonic clonic seizures accompanied by loss of consciousness that lasted for a few seconds. She was admitted at a local hospital and remained well with no recurrence of seizures. She was not maintained on any anti-epileptic medication. However, she was noted to have a weak grip of the right hand described as frequently dropping her milk bottle. Her developmental progress continued to be at par with age.

At 3 years of age, during a bout of fever, the generalized tonic clonic seizures recurred and lasted longer. She was brought to a nearby hospital where status epilepticus was considered. She was prescribed with unrecalled antibiotics and Valproic acid. Cranial CT scan showed a hypodense lesion on the subcortical frontal lobe. She was diagnosed with meningitis and treated with antibiotics.

From 3-5 years of age, the seizures were controlled; however, there was progressive weakening of the grip on both hands and dragging of the left foot on ambulation was noted. She started to have delay in language and personal/social skills. She exhibited hyperactivity and violent behavior described as biting or throwing things at people when she felt frustrated. Cranial magnetic resonance imaging of the brain revealed extensive symmetrical signal abnormalities predominantly involving the subcortical white matter of the cerebral hemispheres bilaterally with accompanying symmetric involvement of the basal ganglia bilaterally, as well as the medial cerebellar hemispheres (Figure 1).

On physical examination at 5 years of age, head circumference was at $85^{\text {th }}-95^{\text {th }}$ percentiles for age. She was not particularly dysmorphic. Neurologic examination showed normal muscle tone, strength and reflexes. There were no tremors. Her gait was noted to be unsteady.

Urinary organic acid was semi-quantitatively analyzed using gas chromatography-mass spectrometry. Internal standards used in the analysis were phenylbutyric acid and tricarballylic acid. Organic acids were extracted by acidification, saturation with salt, and adding ethyl acetate. 

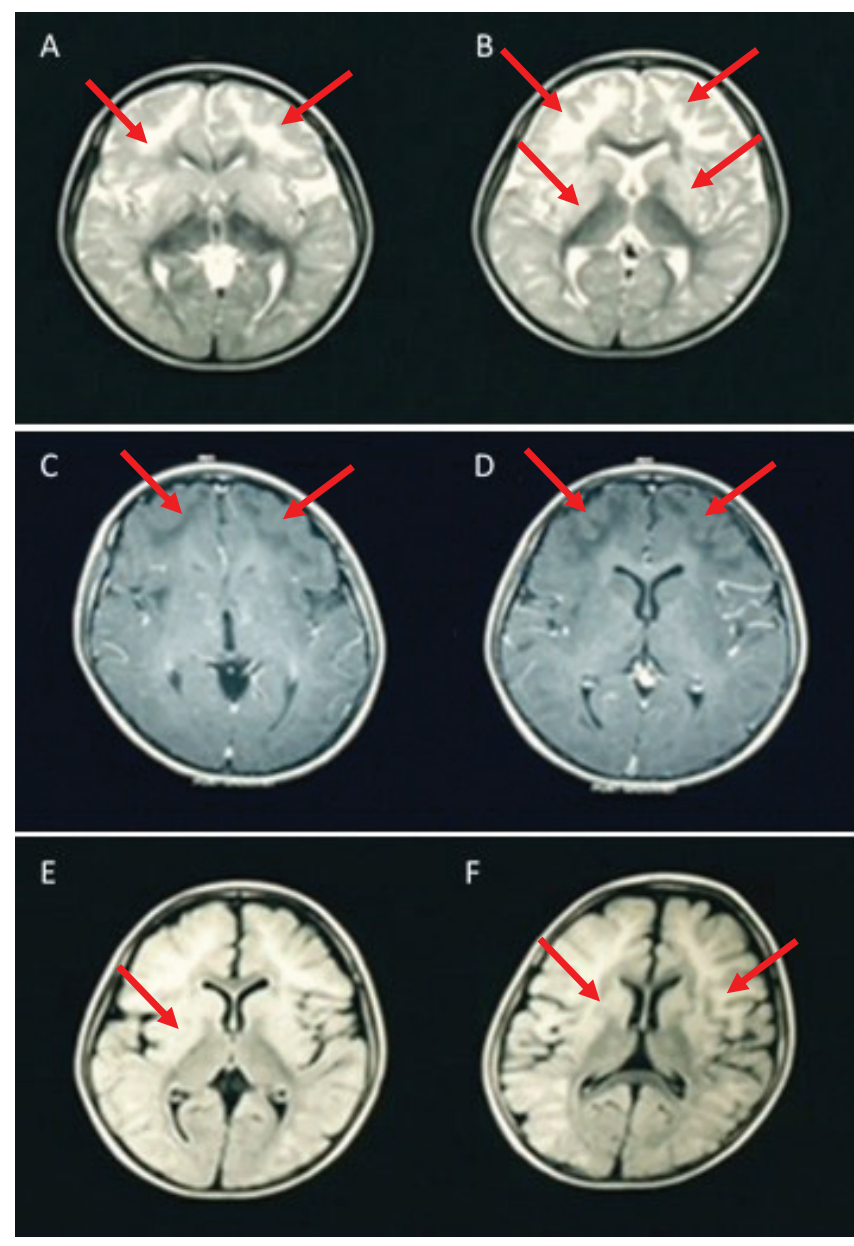

Figure 1. Cranial MRI of the patient. (A, B, C, D) Extensive symmetrical signal abnormalities predominantly involving the subcortical white matter of the cerebral hemispheres bilaterally $(\mathrm{E}, \mathrm{F})$ with accompanying symmetric involvement of the basal ganglia bilaterally.

After separation, sample was dried and derivatized with BSTFA $+1 \%$ trimethylchlorosilane and injected for analysis. Result showed moderately increased level of 2hydroxyglutaric acid (Figure 2).

The enantiomeric determination of L-2-Hydroxyglutaric acid was done at Murdoch Research Institute in Australia using liquid chromatography-tandem mass spectrometry, which revealed gross excretion of L-2-hydroxyglutaric acid at $1470 \mathrm{umol} / \mathrm{mmol}$ of creatinine (normal range 119umol/mmol creatinine).

\section{Discussion}

L-2-HGA is a rare organic aciduria that is inherited in an autosomal recessive pattern. The conversion of L-2hydroxyglutaric acid to alphaketoglutarate is catalyzed by the enzyme L-2-hydroxyglutarate dehydrogenase (L2HGD) with FAD as cofactor. ${ }^{10}$ Molecular studies has showed that mutations in the $\mathrm{L} 2 \mathrm{HGDH}$ gene results to impaired enzyme activity of L-2-hydroxyglutarate dehydrogenase leading to accumulation of L-2-hydroxyglutaric acid. ${ }^{11}$ The increased levels of L-2-hydroxyglutaric acid may have a toxic effect to the central nervous system. ${ }^{10}$ Progress in the understanding of the pathophysiology of L-2 hydroxyglutaric aciduria showed spongiosis and vacuolar lesions in mouse models leading to a consideration that it could be a disorder of metabolite repair. ${ }^{12}$

The disorder has a slow progression without specific clinical signs. Thus, there are delays in the diagnosis of some L-2-HGA patients with a mild phenotype. ${ }^{13}$ Our patient's clinical manifestations were compatible with L-2HGA. Clinical history showed that symptoms started at the age of 1 with seizures followed by motor weakness, developmental delay and ataxia. There are few accounts of L-2-HGA in the literature and among those reported include a 15-year-old boy examined due to epilepsy, whose history revealed that the seizures started at the age of 18 months and eventually showed language regression, progressive intellectual deterioration, and worsening gait. ${ }^{3}$ An adult patient on the other hand was reported to show moderate to severe mental retardation, ataxia, distal tremor in the upper limbs with dystonic movement of the right hand, tetrahyperreflexia, and focal motor deficiencies. ${ }^{14}$

The patient's cranial MRI revealed the typical abnormalities seen in L-2-HGA cases. Magnetic resonance imaging studies of L-2-HGA patients showed highly characteristic abnormalities in the subcortical cerebral white matter, signal changes in the putamina and dentate nuclei, and cerebellar atrophy. ${ }^{4,14}$

2-hydroxyglutaric acid is normally found in urine with equal levels of both $\mathrm{L}$ and $\mathrm{D}$ configuration. Two other biochemical variants of 2-hydroxyglutaric aciduria (2HGA) have been identified with regard to elevated levels of the $\mathrm{L}$, D configurations. ${ }^{13,15}$

D-2-hydroxyglutaric aciduria (D-2-HGA) is biochemically characterized by increased D-2hydroxyglutaric acid in urine, plasma, and CSF. D-2-HGA results from mutations in the D2HGDH gene and IDH2 gene. Clinical findings include developmental delay, epilepsy, and hypotonia with cardiomyopathy found to be exclusive to one phenotype. ${ }^{13}$ In the combined D,L-2hydroxyglutaric aciduria (D,L-2-HGA), both the D and L configuration are moderately increased in urine and plasma. D,L-2-HGA clinically presents with severe neonatal epileptic encephalopathy, seizures, and hypotonia and is caused by SLC25A1 gene mutations. ${ }^{16,17}$ Clinical evaluation and brain MRI findings are relevant in the diagnosis of 2HGA. However, enantiomeric differentiation is required for an accurate differential diagnosis of the disorder. ${ }^{13}$

In our patient, diagnosis was confirmed through organic acid analysis, which showed significant elevation of 2-hydroxyglutaric acid, and enantiomeric identification of L2-hydroxyglutaric acid. Without the availability of enzyme 


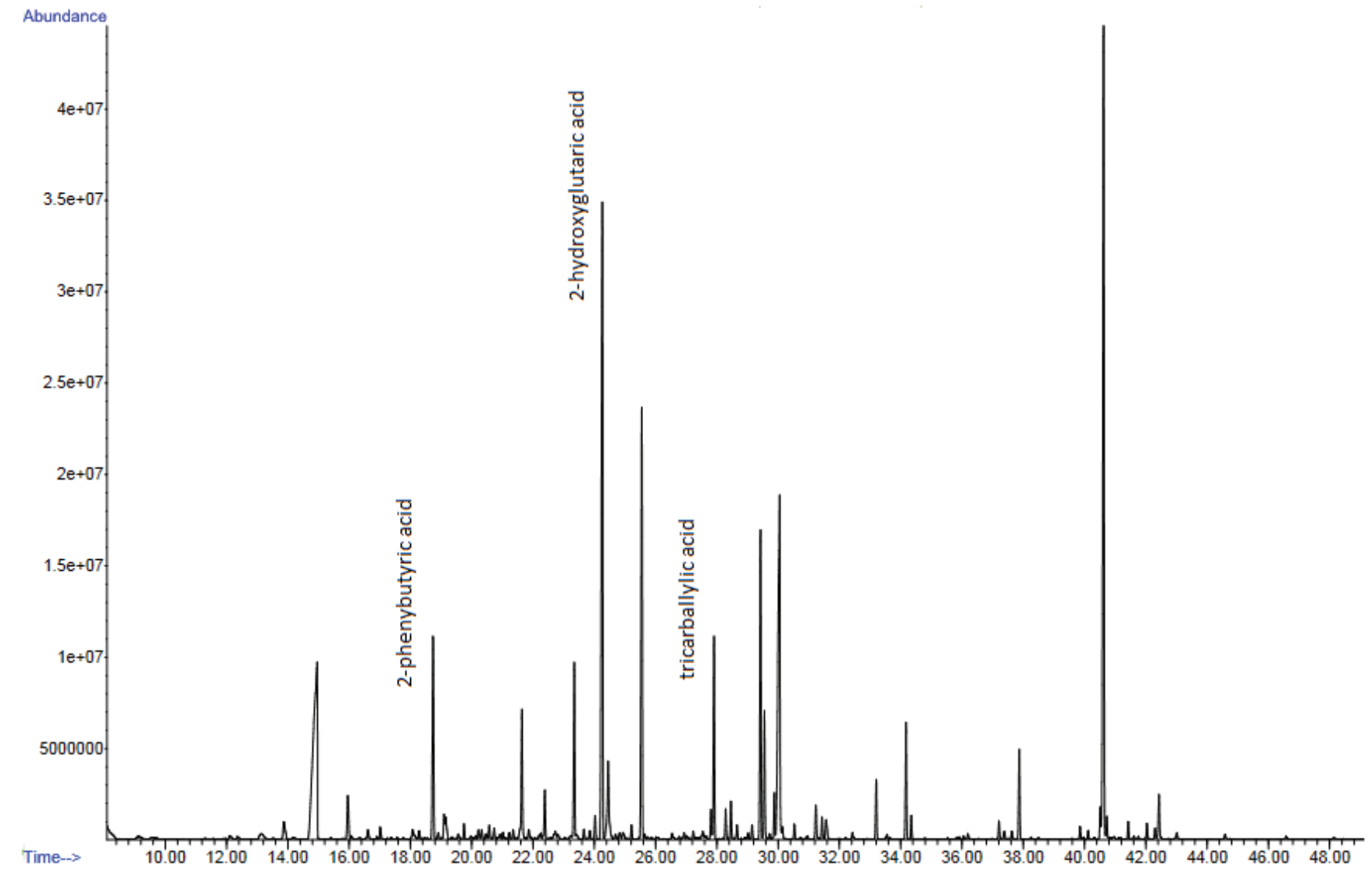

Figure 2. Total ion chromatogram of urinary organic acid metabolites showing increased 2-hydroxyglutaric acid compared to internal standards 2-phenylbutyric and tricarballylic acids.

assays and mutational analysis, the importance of biochemical test for the diagnosis of organic acidurias is reinstated in this report.

Specific therapeutic approaches for L-2-HGA has not yet been established but with L2HGD being FADdependent, supplementation with FAD and riboflavin (precursor of FAD) were investigated for its possible therapeutic role. A patient from Japan was given 30 $\mathrm{mg} /$ day of FAD, which reduced tremor and dystonia gradually and decreased urinary 2-hydroxyglutaric acid. ${ }^{18}$ Treatment with riboflavin $(100 \mathrm{mg} /$ day) of a 16 -year-old patient showed partial improvement in cognitive and motor performances but increasing the dosage showed no further improvement. ${ }^{19}$ In contrast, a report also showed that riboflavin treatment to a 9-year-old patient was unsuccessful without significant reduction in L-2hydroxyglutaric acid, neurological assessment and MRI readings remain unchanged. ${ }^{20}$ These trials show that riboflavin and FAD treatment may only be effective in cases of mild missense mutations in $22 H G D H .{ }^{13}$ Our patient did not receive any medical therapy for L2HGA but continued to receive supportive management for the control of her seizures.

\section{Conclusion}

In summary, the clinical description and biochemical findings in this paper are consistent with L-2hydroxyglutaric aciduria. Baseline urine amino acid and organic screening are helpful tools in diagnosing children with nonspecific neurologic symptoms such as developmental delay, seizures or intellectual impairment.

\section{Acknowledgment}

The authors thank the Section of Neurodevelopmental Pediatrics of the Philippine General Hospital for their assistance.

\section{Statement of Authorship}

All authors have approved the final version submitted.

\section{Author Disclosure}

All authors declared no conflict of interest.

\section{Funding Source}

This paper was funded by the Institute of Human Genetics. 


\section{L-2-Hydroxyglutaric Aciduria}

\section{References}

1. Duran M, Kamerling JP, Bakker, HD, van Gennip AH, Wadman SK. L-2hydroxyglutaric aciduria: an inborn error of metabolism? J. Inherit Metab Dis. 1980; 3(4):109-12.

2. Barth PG, Hoffmann GF, Jaeken J, et al. L-2-hydroxyglutaric acidaemia: clinical and biochemical findings in 12 patients and preliminary report on L-2-hydroxyacid dehydrogenase. J. Inherit Metab Dis. 1993; 16: 75361.

3. Siejo-Martinez M, Navarro C, Castro del Rio M, Vila O, Puig M, Ribes A, Butron M. L-2-hydroxyglutaric aciduria: clinical, neuroimaging, and neuropathological findings. Arch Neurol. 2005; 62(4):666-70.

4. Steenweg ME, Salomons GS, Yapici Z, et al. L-2-hydroxyglutaric aciduria: pattern of MR imaging abnormalities in 56 patients. Radiology. 2009; 251(3):856-65.

5. Aghili M, Zahedi F, Rafiee E. Hydroxyglutaric aciduria and malignant brain tumor: a case report and literature review. J Neurooncol. 2009; 91(2):233-6.

6. Moroni, I, D'Incerti L, Farina L, Rimoldi M, Uziel G. Clinical, biochemical and neuroradiological findings in L-2-hydroxyglutaric aciduria. Neurol Sci. 2000; 21(2):103-8.

7. Fujitake J, Ishikawa Y, Fujii H, et al. L-2-hydroxyglutaric aciduria: two Japanese adult cases in one family. J Neurol. 1999; 246(5):378-82.

8. Clerc C, Bataillard M, Richard P, Divry P, Kraehenbuhl J, Rumbach L. An adult form of L-2-hydroxyglutaric aciduria revealed by tremor. Eur Neurol. 2000; 43(2):119-20.

9. Prevalence of rare diseases: Bibliographic data, Orphanet Report Series, Rare Diseases collection, November 2016, Number 1: Disease listed in alphabetical order. [Online]. 2016 [cited 2017 Feb]. Available from http://www.orpha.net/orphacom/cahiers/docs/GB/Prevalence_of_rare_d iseases_by_diseases.pdf.
10. Rzem R, Veiga-da-Cunha M, Noël G, et al. A gene encoding a putative FAD-dependent L-2-Hydroxyglutarate dehydrogenase is mutated in L2-hydroxyglutaric aciduria. Proc Natl Acad Sci USA. 2004; 101(48):16849-54.

11. Topcu M, Jobard F, Halliez S, et al, L-2-hydroxyglutaric aciduria: identification of a mutant gene C14orf160, localized on chromosome 14q22.1. Hum Mol Genet. 2004; 13(22):2803-11.

12. Rzem R, Achouri Y, Marbaix E, et al. A mouse model of L-2hydroxyglutaric aciduria, a disorder of metabolite repair. PLoS One. 2015; 10 (3):e0119540.

13. Kranendijk M, Struys EA, Salomons GS, Van der Knaap MS, Jakobs C. Progress in understanding 2-hydroxyglutaric acidurias. J Inherit Metab Dis. 2012; 35(4):571-87.

14. Mazzei R, Ungaro C, Garreffa G. Clinical, genetic and magnetic resonance findings in an Italian patient affected by L-2-hydroxyglutaric aciduria. Neurol Sci. 2011; 32:95-99.

15. Muntau A, Röshinger W, Merkenschlager A, et al. Combined D-2- and L-2- hydroxyglutaric aciduria with neonatal onset encephalopathy: third biochemical variant of 2-hydroxyglutaric aciduria Neuropediatrics. 2000; 31:137-40.

16. Nota B, Struys EA, Pop A, et al. Deficiency in SLC25A1, encoding the mitochondrial citrate carrier, causes combined D-2- and L-2 hydroxyglutaric aciduria. Am J Hum Genet. 2013; 92(4):627-31.

17. Mühlhausen C, Salomons GS, Lukacs Z, et al. Combined D2-/L2hydroxyglutaric aciduria (SLC25A1 deficiency): clinical course and effects of citrate treatment. J. Inherit Metab Dis. 2014; 37(5):775-81.

18. Samuraki M, Komai K, Hasegawa Y, et al. A successfully treated adult patient with L-2-hydroxyglutaric aciduria. Neurology. 2008; 70(13):1051-2.

19. Yilmaz K. Riboflavin treatment in a case with L-2-hydroxyglutaric aciduria. Eur J Paediatr Neurol. 2009; 13(1):57-60.

20. Jequier Gygax M, Roulet-Perez E, Meagher-Villemure K, et al. Sudden unexpected death in an infant with L-2-hydroxyglutaric aciduria. Eur J Pediatr. 2009; 168(8):957-62. 